\title{
Genetic Markers for Adolescent Idiopathic Scoliosis on Chromosome 19p13.3 among Saudi Arabian Girls
}

\author{
Abdallah Ahmad Al-Othman ${ }^{1}$, Mir Sadat-Ali ${ }^{1}$, Ahmed Sh. Amer ${ }^{2}$, Dakheel A. Al-Dakheel ${ }^{3}$ \\ ${ }^{1}$ Department of Orthopaedic Surgery, College of Medicine, Imam Abdulrahman Bin Faisal University, \\ Dammam and King Fahd Hospital of the University, Alkhobar, Saudi Arabia \\ ${ }^{2}$ Department of Orthopaedic Surgery, King Fahd Hospital of the University, Alkhobar, Saudi Arabia \\ ${ }^{3}$ Department of Spinal Surgery, College of Medicine, Imam Abdulrahman Bin Faisal University, \\ Dammam and King Fahd Hospital of the University, Alkhobar, Saudi Arabia
}

\begin{abstract}
Study Design: Prospective case-controlled study.
Purpose: This study aimed to assess genetic influence in Saudi Arabian children with adolescent idiopathic scoliosis (AIS).

Overview of Literature: The genetic locus linked to chromosome 19p for idiopathic scoliosis has been described. A pilot study conducted at King Fahd Hospital of the University, Al-Khobar showed that three microsatellite markers (D19S216, D19S894, and DS1034) of chromosome 19p13.3 were significant in Saudi Arabian females compared with healthy subjects.

Methods: A total of 100 unrelated Saudi Arabian girls treated for AIS, their parents, healthy siblings, and healthy subjects were recruited for genetic analysis of markers on chromosome 19p13.3. After informed consent was obtained from their parents, blood samples were collected and parametric and nonparametric linkage analyses were performed using GENEHUNTER ver. 2.1. Multipoint linkage analysis was used to specify an autosomal dominant trait with a gene frequency of 0.01 and an estimated penetrance of $80 \%$ at the genotypic and allelic levels.

Results: Five hundred blood samples were collected and analyzed for microsatellite markers (D19S216, D19S894, and DS1034) of chromosome 19p13.3. Comparison among patients, family members, and healthy subjects revealed no significant association between markers and scoliosis at the genotypic level: D19S216 ( $p=0.21)$, D19S894 ( $p=0.37)$, and DS1034 ( $p=0.25)$. However, at the allelic level, a statistically significant association was observed for marker DS1034 ( $p=0.008)$, and marker D19S216 showed significance between fathers and patients $(p<0.001)$ compared with patients and mothers. The other two markers, D19S216 ( $p=0.25)$ and D19S894 ( $p=0.17)$, showed no significant association between patients and mothers.

Conclusions: At the allelic level, marker DS1034 was significantly associated with AIS patients and their fathers. This allelic marker on chromosome 19p13.3 appears to be important in AIS etiology.
\end{abstract}

Keywords: Adolescent idiopathic scoliosis; Chromosome 19p13.3; Saudi Arabia

\footnotetext{
Received Jun 22, 2016; Revised Aug 26, 2016; Accepted Sep 2, 2016

Corresponding author: Mir Sadat-Ali

Department of Orthopaedic Surgery, College of Medicine, Imam Abdulrahman Bin Faisal University, Dammam and King Fahd Hospital of the University, P.O. Box 40071, Al-Khobar 31952, Saudi Arabia

Tel: +96-6138820887, Fax: +96-6138820887, E-mail: drsadat@hotmail.com
} 


\section{Introduction}

Scoliosis is one of the most common spinal deformities, occurring $2 \%-4 \%$ of school-aged children [1]. Idiopathic scoliosis (IS) occurs without any known cause in otherwise healthy children and spares no ethnic group [2]. Girls are more at risk for progression by a ratio of 3.6:1 [3]; however, the reason for boys having less progression remains unclear [4]. The incidence of scoliosis in Saudi Arabia was reported to be $0.16 \%-0.5 \%[5,6]$, with $59 \%$ of those cases being IS [7]. Because McCarthy [8] suggested that complications in scoliosis can be prevented by early diagnosis and appropriate treatment, school screening was established for the early diagnosis of scoliosis to prevent complications of late presentation. The reported complications of scoliosis surgery are serious [9] and can be avoided in a majority of children with IS if early diagnosis and proper bracing is used. In conservative societies, girls often remain covered, and early spine deformities are easily missed such that most of the affected children end up being treated operatively There is currently no clear consensus regarding the genetic influence on scoliosis; however, reports indicate that adolescent IS (AIS) is linked to a few chromosomes [10-15], and most of these linkages are found to be in familial scoliosis. Heary and Madhavan [16] stated that scoliosis can be inherited because of autosomal dominant, X-linked, or multi-gene influences. Chan et al. [17] found a genetic locus linked to chromosome 19p13.3 in Asian families of patients with AIS and defined AIS in the critical region within the vicinity of D119S216, D19S894, and D19S1034, which was further confirmed by Alden et al. [18]. A pilot study conducted in Saudi Arabia showed that a significant difference existed between the genetic markers in Saudi Arabian girls with AIS and healthy subjects [19]. Both studies had small numbers of patients in the study and control groups. This study aimed to identify genetic markers on chromosome 19p13.3 in Saudi Arabian girls with AIS and compare these markers with those of a control group.

\section{Materials and Methods}

The study was initiated after approval from the Institutional Review Board of the University of Dammam. A search through the hospital Ulticare system identified patients from the outpatient department who visited/were operated for AIS. Patients with a Cobb angle of $\geq 30^{\circ}$ were referred to the outpatient clinics, and an informed consent was signed by their parents after explaining the study. Demographic data were collected and entered into the database. Blood samples were collected from 100 patients, 200 parents, and 100 unaffected siblings. Blood samples for the control group were obtained from 100 patients who had consented to blood tests for another study.

The samples were processed, and genotyping was performed using Sequencing, SNaPshot, TaqMan, Allele Specific Amplification, and Cleaved Amplified Polymorphic Sequence. DNA extraction was performed on the blood samples, and genotyping was performed using TaqMan OpenArray (ThermoFisher Scientific, Waltham, MA, USA) Format 16 assays. Sample mixes were loaded onto a TaqMan OpenArray Genotyping Plate, which was then inserted into the TaqMan OpenArray case filled with immersion fluid and sealed. Plate cycling was performed using a qualified thermal cycler, and plate imaging was performed using the OpenArray NT Imager. Data extraction and SNP analysis were performed, and the data were entered into the database. Statistical analyses of contingency tables for D19S894, D10S216, and D19S1034 were performed using Fisher exact test, with $p<0.05$ being con-

Table 1. Demographic data of affected subjects

\begin{tabular}{|c|c|c|c|c|c|}
\hline No. of patients & $\begin{array}{c}\text { Age at presentation } \\
\text { (yr) }\end{array}$ & Site of deformity & $\begin{array}{l}\text { Average Cobb } \\
\text { angle }\left({ }^{\circ}\right)\end{array}$ & $\begin{array}{l}\text { Brace prior } \\
\text { to surgery }\end{array}$ & Surgery \\
\hline 22 & $14-16$ & $\begin{array}{l}\mathrm{TL}(18) \\
\mathrm{T}(4)\end{array}$ & $\begin{array}{l}54.6 \\
45\end{array}$ & $\begin{array}{l}2 \\
2\end{array}$ & $\begin{array}{r}16 \\
2\end{array}$ \\
\hline 37 & $17-19$ & $\begin{array}{l}\mathrm{TL}(32) \\
\mathrm{T}(5)\end{array}$ & $\begin{array}{l}48.5 \\
42\end{array}$ & $\begin{array}{l}5 \\
3\end{array}$ & $\begin{array}{r}21 \\
2\end{array}$ \\
\hline 41 & $\geq 20$ & $\begin{array}{c}\mathrm{TL}(27) \\
\mathrm{T}(9) \\
\mathrm{L}(5)\end{array}$ & $\begin{array}{l}55.2 \\
47 \\
38\end{array}$ & $\begin{array}{l}0 \\
0 \\
2\end{array}$ & $\begin{array}{r}27 \\
9 \\
3\end{array}$ \\
\hline
\end{tabular}

T, thoracic; $T L$, thoraco-lumbar; L, lumbar curves. 
sidered significant.

\section{Results}

A total of 464 samples were obtained for analysis. There were 82 female and 18 male patients with AIS, with an average age of $18.52 \pm 3.6$ years. Table 1 shows the demographic data for all patients, and Table 2 shows the summary of microsatellite markers of chromosome 19p13.3. The three microsatellite markers with their allelic IDs and frequencies were studied on chromosome 19p13.3 (Table 2). Fig. 1 shows that no significant linkage difference was observed between the markers and scoliosis using a modelbased approach. All nonparametric logarithm (base 10) of odds scores were equal to 0 . Table 3 shows that there was no statistical difference at the genetic level between fathers and mothers compared with patients $(p<0.1$ and $p<0.5)$ for marker D19S894. For marker D19S216, a significant difference was observed between mothers and patients $(p<0.08)$ (Table 4$)$ while in comparison the genetic distribution of fathers and patients were similar $(p<0.11)$. With regard to marker D19S1034, no significant difference was observed among patients, mothers, and fathers (mothers vs. patients $[p<0.085]$ and fathers vs. patients $[p<0.5])$ (Table 5). At the allelic level, no statistical significance was observed among patients, mothers, and fathers for marker D19S894 (mothers vs. patients [ $p<0.1]$ and fathers vs. patients $[p<0.5])$ (Table 5). The analysis of marker D19S216 at the allelic level showed significance between fathers and

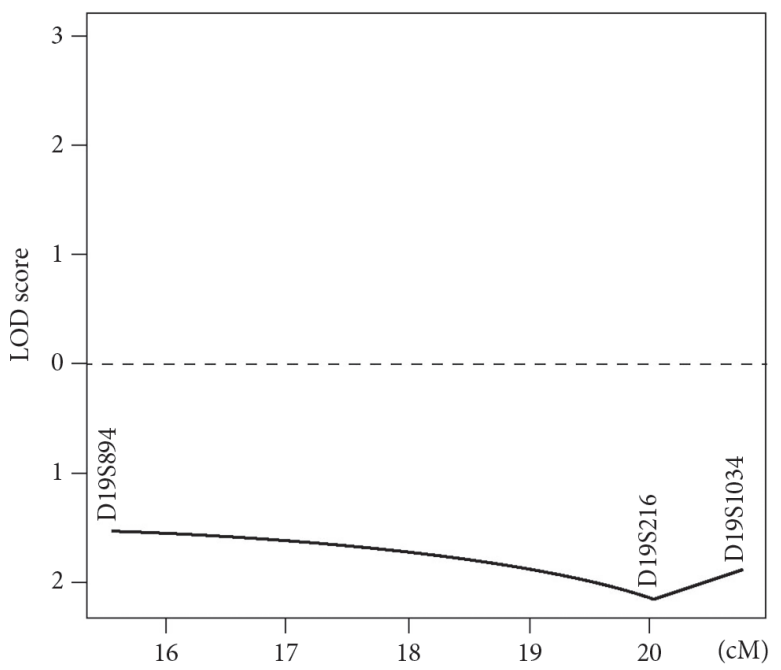

Fig. 1. Logarithm (base 10) of odds (LOD) scores with linkage distances.

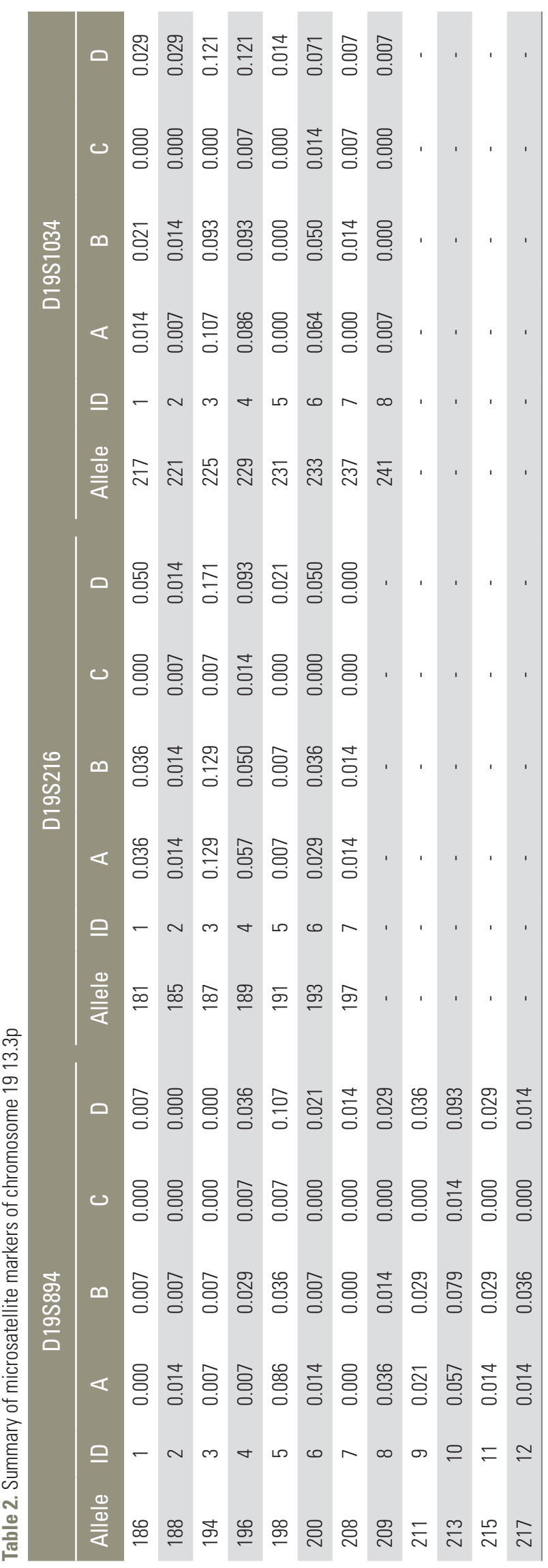


Table 3. Analysis at genotypic level (healthy patient)

D19S894 ( $p=0.731)$

\begin{tabular}{|c|c|c|c|c|c|c|c|c|c|c|c|c|c|c|c|c|}
\hline 111 & 211 & 26 & 24 & 312 & 49 & 44 & 411 & 410 & 45 & 55 & 513 & 510 & 511 & 512 & 58 & 56 \\
\hline 1 & 1 & 1 & 1 & 2 & 1 & 1 & 0 & 1 & 1 & 3 & 1 & 5 & 0 & 1 & 0 & 1 \\
\hline 1 & 0 & 0 & 0 & 0 & 1 & 0 & 2 & 1 & 1 & 1 & 1 & 5 & 1 & 2 & 1 & 1 \\
\hline 59 & 67 & 68 & 610 & 710 & 810 & 811 & 89 & 88 & 910 & 99 & 1014 & 1010 & 1011 & 1012 & 1112 & 1212 \\
\hline 3 & 0 & 1 & 0 & 0 & 1 & 2 & 1 & 1 & 2 & 0 & 2 & 4 & 1 & 1 & 1 & 1 \\
\hline 1 & 1 & 0 & 1 & 1 & 3 & 0 & 0 & 0 & 1 & 1 & 1 & 0 & 0 & 0 & 0 & 0 \\
\hline
\end{tabular}

Table 4. The contingency table below showing analysis of marker D19S216 at genotypic level

\begin{tabular}{llllllllllllllll} 
Subjects tested & 16 & 14 & 13 & 15 & 23 & 24 & 36 & 33 & 35 & 34 & 37 & 47 & 44 & 66 \\
Mother & 1 & 0 & 4 & 0 & 2 & 0 & 3 & 2 & 2 & 3 & 1 & 1 & 2 & 0 \\
Patient & 0 & 2 & 5 & 0 & 2 & 0 & 3 & 3 & 3 & 5 & 0 & 0 & 3 & 2 \\
Father & 0 & 1 & 3 & 1 & 2 & 0 & 3 & 2 & 0 & 4 & 2 & 0 & 1 & 1 \\
\hline
\end{tabular}

$p<0.08$ (mother and patients) and $p<0.11$ (father and patients).

Table 5. The contingency table below showing analysis of marker D19S1034 at genotypic level

\begin{tabular}{lrrrrrrrrrrrrrrrrr}
$\begin{array}{l}\text { Subjects } \\
\text { tested }\end{array}$ & 15 & 13 & 14 & 24 & 27 & 23 & 33 & 34 & 37 & 36 & 35 & 38 & 46 & 44 & 47 & 66 \\
Mother & 0 & 10 & 0 & 0 & 0 & 5 & 10 & 20 & 0 & 15 & 0 & 5 & 20 & 10 & 0 & 5 \\
Patient & 10 & 15 & 5 & 5 & 5 & 10 & 5 & 20 & 5 & 5 & 3 & 5 & 3 & 4 & 0 & 0 \\
Father & 0 & 10 & 5 & 5 & 0 & 5 & 5 & 10 & 10 & 20 & 0 & 0 & 15 & 15 & 0 & 0 \\
\hline
\end{tabular}

Mothers versus patients $p=0.0852$ and fathers versus patients $p<0.5$.

Table 6. Contingency table below showing analysis of D19S894 at allele level

\begin{tabular}{lcccccccccccccc} 
Subjects tested & 1 & 2 & 3 & 4 & 5 & 6 & 7 & 8 & 9 & 10 & 11 & 12 & 13 & 14 \\
Mother & 0 & 6 & 8 & 8 & 24 & 4 & 0 & 10 & 6 & 16 & 5 & 3 & 6 & 4 \\
Patient & 10 & 0 & 0 & 10 & 16 & 12 & 8 & 8 & 10 & 10 & 8 & 4 & 3 & 1 \\
Father & 12 & 2 & 2 & 10 & 10 & 2 & 8 & 4 & 8 & 22 & 8 & 10 & 0 & 2 \\
\hline
\end{tabular}

Mothers versus patients $p<0.1$ and fathers versus patients $p<0.5$.

Table 7. Contingency table showing analysis of marker D19S216 at allele level

\begin{tabular}{lccccccc} 
Subjects tested & 1 & 2 & 3 & 4 & 5 & 6 & 7 \\
Mother & 16 & 18 & 20 & 15 & 15 & 8 & 12 \\
Father & 10 & 14 & 22 & 18 & 10 & 14 & 12 \\
\hline Patient & 10 & 12 & 24 & 20 & 12 & 10 \\
\hline
\end{tabular}

Mothers versus patients $p<0.3$ and fathers versus patients $p<0.001$.

patients $(p<0.001)$ compared with patients and mothers $(p<0.3)$ (Table 6). Analysis of marker D19S216 at the allelic level between mothers and patients resulted in $p<0.5$ and was statistically significant between patients and fa- thers $(p<0.001)$ (Table 7). Analysis of marker DS19S1034 was similar between fathers and patients and was significant between mothers and patients (Table 8). Fig. 2 show the radiograph of the thoraco-lumbar spine of patients 
Table 8. Contingency table showing analysis of marker D19S1034 at allele level

\begin{tabular}{lcccccccc} 
Subjects tested & 1 & 2 & 3 & 4 & 5 & 6 & 7 & 8 \\
Mother & 8 & 8 & 15 & 12 & 10 & 10 & 7 & 10 \\
Father & 8 & 8 & 14 & 14 & 8 & 10 & 8 & 10 \\
\hline Patient & 10 & 8 & 22 & 24 & 14 & 10 & 12 & 12 \\
\hline
\end{tabular}

Mothers versus patients $p<0.04$ and fathers versus patients $p<0.1$.

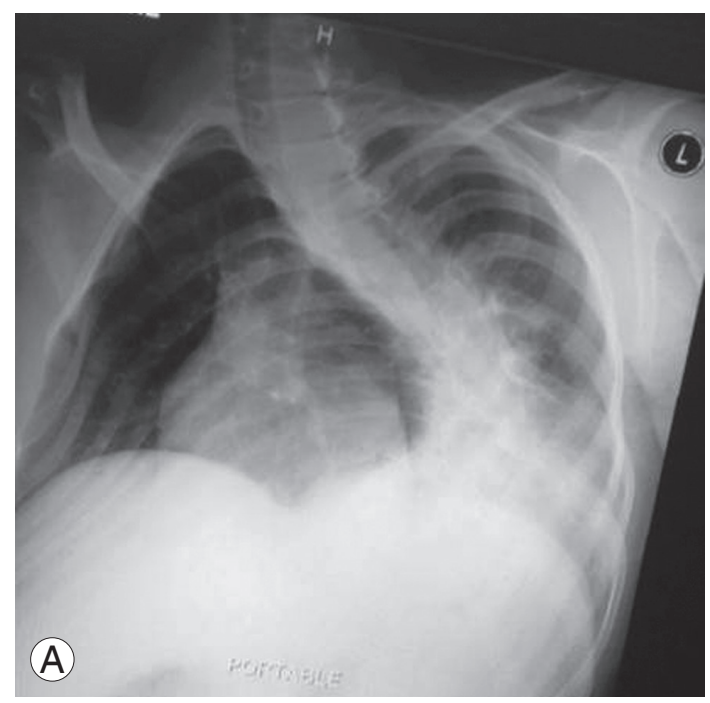

Fig. 2. (A) A 20-year-old girl who was one of the screened patients that presented to the hospital with severe deformity 3 months before. Because all females are completely covered with a dress, the deformity was missed, and the patient never complained to her parents. The genetic tests showed that at the allelic level, a statistically significant association existed for markers DS1034 and D19S216 between the father and patient. (B) The spinal radiograph of the first cousin of the patient is given above. The allele makeup was similar to that of the father and different from those of the mother and sibling.

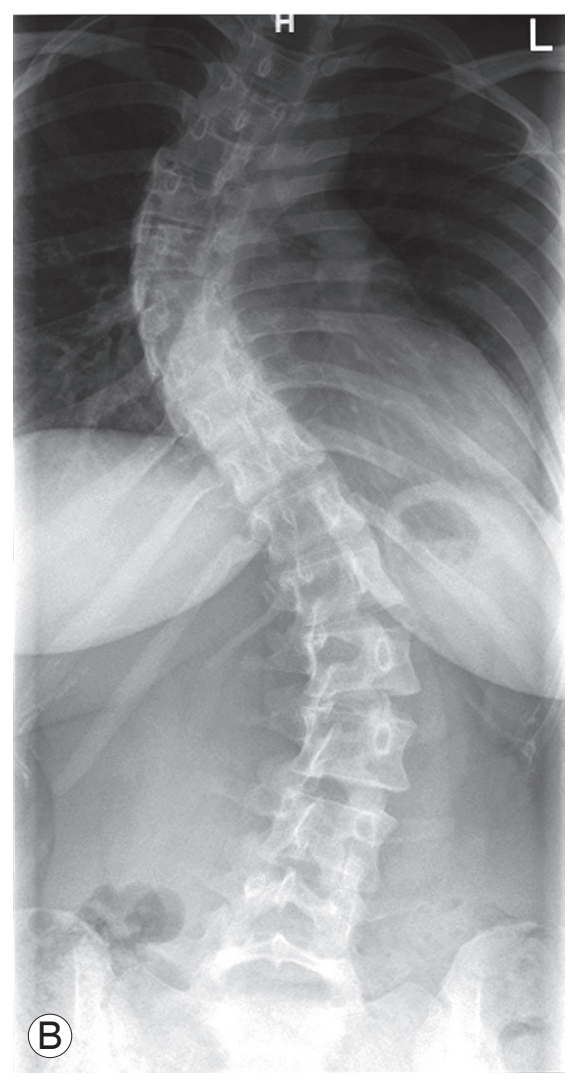

with familial AIS where the allele was similar to that of the father.

\section{Discussion}

This study demonstrated a linkage between fathers and patients at the genotypic level of marker D19S216, whereas the other two markers were not significantly similar. At the allelic level of marker D19S216, the alleles of the mothers were similar to those of the patients, whereas for the fathers, it was significant $(p<0.001)$ in patients with AIS on chromosome 19p13.3 among all ethnic Saudi Arabian boys and girls. The three markers were present on chromosome 19p13.3 at a distance spanning $5.2 \mathrm{cM}$. The results of this study were similar to those of an ear- lier study with regard to the comparison among patients, siblings, and controls [19]. The controls included unrelated healthy individuals who were unaffected and who showed no similarity with the patients at the allelic and genotypic levels. Chan et al. [17] reported that in Chinese families with AIS, a genetic locus was found to be linked to chromosome 19p13.3 within the vicinity of markers D119S216, D19S894, and D19S1034. Our study results support those reported by Chan et al. [17], which showed that a definite linkage to chromosome 19p3.3 exits and that it is related to only one marker D119S216. However, this differs between the mothers and fathers at the genotypic and allelic levels.

Many other chromosomes were reported to carry markers that influenced familial AIS. Chromosomes previously 
studied included $6,9,12,16$, and 17 . Most of the studies regarding genetic influence on AIS were conducted in families: chromosomes 6p (D6S1051-D6S1017), 6q (D6S1053-D6S1021), 8q IS3 (D8S1477-D8S279), 9q (D9S938-D9S934), 10q (D10S1222-D10S212), 16q (D16S764-D16S2624), 17p IS2 (D17S974-D17S1294), 18q (D18S1357-D18S1371), and 19p IS1 (D19S1034) $[10,11,13-15,17,18]$. Our study was performed in patients who were unrelated, and we found a risk for one marker. $\mathrm{Xu}$ et al. [20] recently reported a case-control study that genotyped twenty SNPs of the GPR126 gene in unrelated AIS patients. They further added 10 exonic SNPs and 10 intronic polymorphisms in patients and compared them to those in controls, finding strong evidence in $30 \%$ of SNPs studied. They concluded that genetic variants of the GPR126 gene are associated with AIS susceptibility.

The scientific literature suggests that AIS is a disease with polygenic inheritance because the low penetrance of its alleles leads to different expressions [21]. For a long time, it was believed that the inheritance was a result of autosomal dominant [22-24] and because of a lack of male to female transmissions [25]. In our study, we found a similarity between patients and fathers rather than between patients and mothers at the genetic level. Haller et al. [25] suggested that AIS inheritance follows a complex genetic architecture with a polygenic pattern of inheritance. These new findings may be consistent with a multifactorial inheritance model, with many genes and diseases being modified by environmental influences. This study aimed to confirm the link in a larger group of patients to obtain a clear statistical significance, which we could confirm. This will help in screening suspected patients early in life so that bracing may limit the progression of the disease.

A limitation of this study is that we screened patients from only one region of the country. Nonetheless, a strength of the study is that the patient population was 100 , which is a good number. Another strong point is that we compared mothers, fathers, siblings, and unrelated and unaffected controls, which gave some indication of the transmission of the disease from fathers to offspring.

\section{Conclusions}

Our study indicates that of the markers of chromosome 19 p13.3 studied, D19S216 was associated with a high risk for AIS, and the inheritance appears to be autosomal dominant. We believe that more genomic studies are required to discover more genetic markers, which could thoroughly describe AIS susceptibility. Appropriate genetic screening will enable early diagnosis of AIS, leading to less invasive therapies in AIS management.

\section{Conflict of Interest}

No potential conflict of interest relevant to this article was reported.

\section{Acknowledgments}

The authors thank Deanship of Scientific Research, Imam Abdulrahman Bin Faisal University, Dammam for funding this project.

\section{References}

1. Lonstein JE. Idiopathic scoliosis. In: Lonstein JE, Bradford DS, Winter RB, Ogilive JW, editors. Moe's textbook of scoliosis and other spinal deformities. 3rd ed. Philadelphia: WB Saunders; 1995. p.219-56.

2. Herring JA. Tachdjian's pediatric orthopaedics. Philadelphia: WB Saunders; 2002.

3. Weinstein SL. Adolescent idiopathic scoliosis: prevalence natural history. In: Weinstein SL, editor. The pediatric spine: principle and practice. New York: Raven Press; 1994. p.463-78.

4. Karol LA, Johnston CE 2nd, Browne RH, Madison M. Progression of the curve in boys who have idiopathic scoliosis. J Bone Joint Surg Am 1993;75:1804-10.

5. Corea JR, Magbool GM, Al Arfag A, Sankarankutty M. School screening for scoliosis in Saudi Arabia. Saudi Med J 1993;14:209-12.

6. Sadat-Ali M. School screening for scoliosis: have we done enough? Saudi Med J 1998;19:210-1.

7. Al-Arjani AM, Al-Sebai MW, Al-Khawashki HM, Saadeddin MF. Epidemiological patterns of scoliosis in a spinal center in Saudi Arabia. Saudi Med J 2000; 21:554-7.

8. McCarthy RE. Prevention of the complications of scoliosis by early detection. Clin Orthop Relat Res 1987;(222):73-8.

9. Weiss HR, Goodall D. Rate of complications in scoliosis surgery: a systematic review of the Pub Med literature. Scoliosis 2008;3:9. 
10. Wise CA, Barnes R, Gillum J, Herring JA, Bowcock AM, Lovett M. Localization of susceptibility to familial idiopathic scoliosis. Spine (Phila Pa 1976) 2000;25: 2372-80.

11. Salehi LB, Mangino M, De Serio S, et al. Assignment of a locus for autosomal dominant idiopathic scoliosis (IS) to human chromosome 17p11. Hum Genet 2002;111:401-4.

12. Ocaka L, Zhao C, Reed JA, et al. Assignment of two loci for autosomal dominant adolescent idiopathic scoliosis to chromosomes 9q31.2-q34.2 and 17q25.3qtel. J Med Genet 2008;45:87-92.

13. Justice CM, Miller NH, Marosy B, Zhang J, Wilson AF. Familial idiopathic scoliosis: evidence of an Xlinked susceptibility locus. Spine (Phila Pa 1976) 2003;28:589-94.

14. Miller NH, Justice CM, Marosy B, et al. Identification of candidate regions for familial idiopathic scoliosis. Spine (Phila Pa 1976) 2005;30:1181-7.

15. Gao X, Gordon D, Zhang D, et al. CHD7 gene polymorphisms are associated with susceptibility to idiopathic scoliosis. Am J Hum Genet 2007;80:957-65.

16. Heary RF, Madhavan K. Genetics of scoliosis. Neurosurgery 2008;63(3 Suppl):222-7.

17. Chan V, Fong GC, Luk KD, et al. A genetic locus for adolescent idiopathic scoliosis linked to chromosome 19p13.3. Am J Hum Genet 2002;71:401-6.

18. Alden KJ, Marosy B, Nzegwu N, Justice CM, Wilson
AF, Miller NH. Idiopathic scoliosis: identification of candidate regions on chromosome 19p13. Spine (Phila Pa 1976) 2006;31:1815-9.

19. Sadat-Ali M, Al-Omran AS, Al-Othman AA. Genetic markers for idiopathic scoliosis on chromosome 19p 13.3 among Saudi Arabian girls: a pilot study. Indian J Hum Genet 2011;17:13-6.

20. Xu JF, Yang GH, Pan XH, et al. Association of GPR126 gene polymorphism with adolescent idiopathic scoliosis in Chinese populations. Genomics 2015;105:101-7.

21. Ward K, Ogilvie J, Argyle V, et al. Polygenic inheritance of adolescent idiopathic scoliosis: a study of extended families in Utah. Am J Med Genet A 2010; 152:1178-88.

22. Bell M, Teebi AS. Autosomal dominant idiopathic scoliosis? Am J Med Genet 1995;55:112.

23. Cowell HR, Hall JN, MacEwen GD. Genetic aspects of idiopathic scoliosis: a Nicholas Andry Award essay, 1970. Clin Orthop Relat Res 1972;86:121-31.

24. Riseborough EJ, Wynne-Davies R. A genetic survey of idiopathic scoliosis in Boston, Massachusetts. J Bone Joint Surg Am 1973;55:974-82.

25. Haller G, Alvarado D, McCall K, et al. A polygenic burden of rare variants across extracellular matrix genes among individuals with adolescent idiopathic scoliosis. Hum Mol Genet 2016;25:202-9. 